\title{
Approximation of Durrmeyer Type Operators Depending on Certain Parameters
}

\author{
Neha Malik, ${ }^{1}$ Serkan Araci, ${ }^{2}$ and Man Singh Beniwal ${ }^{3}$ \\ ${ }^{1}$ Department of Mathematics, Netaji Subhas Institute of Technology, Sector 3 Dwarka, New Delhi 110078, India \\ ${ }^{2}$ Faculty of Economics, Social and Administrative Sciences, Hasan Kalyoncu University, 27410 Gaziantep, Turkey \\ ${ }^{3}$ Department of Applied Sciences, Maharaja Surajmal Institute of Technology, C-4 Janak Puri, New Delhi 110058, India \\ Correspondence should be addressed to Serkan Araci; mtsrkn@hotmail.com
}

Received 29 January 2017; Revised 30 March 2017; Accepted 30 April 2017; Published 30 May 2017

Academic Editor: Changbum Chun

Copyright (C) 2017 Neha Malik et al. This is an open access article distributed under the Creative Commons Attribution License, which permits unrestricted use, distribution, and reproduction in any medium, provided the original work is properly cited.

Motivated by a number of recent investigations, we consider a new analogue of Bernstein-Durrmeyer operators based on certain variants. We derive some approximation properties of these operators. We also compute local approximation and Voronovskaja type asymptotic formula. We illustrate the convergence of aforementioned operators by making use of the software MATLAB which we stated in the paper.

\section{Dedicated to Professor Vijay Gupta}

\section{Introduction}

In approximation theory, the use of quantum calculus $(q-$ calculus) has gained momentum in the last decade. In the year 1987, Lupaş [1] pioneered the work on $q$-versions of the Bernstein polynomials. After ten years, Phillips gave another $q$ variant of Bernstein polynomials. Since then, numerous operators have been generalized to their quantum variants and their approximation behaviours have been studied; we indicate the recent books $[2,3]$ on this topic. Also, see [4-6]. Lately, the further generalization of $q$-calculus, namely, the postquantum calculus, symbolized by $(p, q)$-calculus has become very contributing. In [7], Mursaleen et al. proposed the $(p, q)$-variant of Bernstein polynomials, which was further improved in [8]. Further, generalizations of $(p, q)$-Bernstein polynomials are due to Kantorovich and Durrmeyer, which were, respectively, studied in $[9,10]$. Many papers pertaining to approximation theory and special functions have been presented recently (cf. [11-21]). The first $q$-variant and $(p, q)$-variant of Bernstein-Durrmeyer operators were given in [22] and [10], respectively. For postquantum calculus, some basic theorems and definitions are as follows (cf. [10, 2325]).
The $(p, q)$-number is defined as

$$
[r]_{p, q}=\frac{p^{r}-q^{r}}{p-q}, \quad p, q \in(0,1] .
$$

It has been observed that $[r]_{p, q}=p^{r-1}[r]_{q / p}$. By this identity, the results obtained in $(p, q)$-calculus cannot be obtained directly from $q$-calculus.

The $(p, q)$-binomial coefficient is known as

$$
\left[\begin{array}{l}
r \\
k
\end{array}\right]_{p, q}=\frac{[r]_{p, q} !}{[r-k]_{p, q} ![k]_{p, q} !}, \quad(0 \leqslant k \leqslant r),
$$

where

$$
[r]_{p, q} !=\prod_{k=1}^{r}[k]_{p, q}, \quad r \geqslant 1,[0]_{p, q} !=1 .
$$

The $(p, q)$-analogue of $(x-\omega)^{r}$ is defined by

$$
\begin{aligned}
& (x \ominus \omega)_{p, q}^{r}=(x-\omega) \\
& \quad \cdot(p x-q \omega)\left(p^{2} x-q^{2} \omega\right) \cdots\left(p^{r-1} x-q^{r-1} \omega\right) .
\end{aligned}
$$


The following $\Gamma_{p, q}(r+1)$

$$
\Gamma_{p, q}(r+1)=\frac{(p \ominus q)_{p, q}^{r}}{(p-q)^{r}}=[r]_{p, q} !,
$$

$$
(0<q<p, r \geq 0)
$$

is $(p, q)$-analogue of Gamma function.

The $(p, q)$-analogue of derivative of a function $f$ is defined by

$$
D_{p, q} f(x)=\frac{f(p x)-f(q x)}{(p-q) x}, \quad(x \neq 0) .
$$

In the case when $p=1$, the $(p, q)$-derivative reduces to known $q$-derivative. Like $q$-derivative, $(p, q)$-derivative has the following properties which are product rules for two functions:

$$
\begin{aligned}
D_{p, q}(f(x) g(x))= & f(p x) D_{p, q} g(x) \\
& +g(q x) D_{p, q} f(x), \\
D_{p, q}(f(x) g(x))= & g(p x) D_{p, q} f(x) \\
& +f(q x) D_{p, q} g(x) .
\end{aligned}
$$

Let $f$ be an arbitrary function and $\omega \in \mathbb{R}$. The $(p, q)$ integral of $f(x)$ on $[0, \omega]$ (see [24]) is defined as

$$
\begin{aligned}
& \int_{0}^{\omega} f(x) d_{p, q} x=(q-p) \omega \sum_{k=0}^{\infty} \frac{p^{k}}{q^{k+1}} f\left(\frac{p^{k}}{q^{k+1}} \omega\right), \\
& \text { if }|p|<|q|, \\
& \int_{0}^{\omega} f(x) d_{p, q} x=(p-q) \omega \sum_{k=0}^{\infty} \frac{q^{k}}{p^{k+1}} f\left(\frac{q^{k}}{p^{k+1}} \omega\right), \\
& \text { if }|q|<|p| .
\end{aligned}
$$

Let $m, n \in \mathbb{N}$, the $(p, q)$-Beta function of first kind be given by

$$
B_{p, q}(m, n)=\int_{0}^{1}(p x)^{m-1}(p \ominus p q x)_{p, q}^{n-1} d_{p, q} x
$$
[10])

The $(p, q)$-Gamma and $(p, q)$-Beta functions satisfy (see

$$
B_{p, q}(m, n)=p^{[n(2 m+n-2)+n-2] / 2} \frac{\Gamma_{p, q}(m) \Gamma_{p, q}(n)}{\Gamma_{p, q}(m+n)},
$$

where $m, n \in \mathbb{N}$.

In the present article, we extend the studies of [10] and discuss their ordinary approximation properties. These include direct estimates in terms of modulus of smoothness and Voronovskaja type asymptotic formula. Moreover, the convergence behaviour is shown graphically using MATLAB.

\section{2. $(p, q)$-Durrmeyer-Stancu Operators and Moments}

The $q$-Durrmeyer operators were considered in [22] and also studied in [26]. Inspired by the work of [10, 27], we consider the postquantum Stancu variant of the eminent Durrmeyer operators. The $(p, q)$-analogue of Durrmeyer-Stancu operators for $x \in[0,1], 0 \leqslant \alpha \leqslant \beta$ and $0<q<p \leqslant 1$ is defined as

$$
\begin{gathered}
D_{n, \alpha, \beta}^{p, q}(f, x)=[n+1]_{p, q} \sum_{k=0}^{n} p^{-\left(n^{2}+3 n-k^{2}-k\right) / 2} b_{n, k}^{p, q}(1, x) \\
\cdot \int_{0}^{1} b_{n, k}^{p, q}(p, p q t) f\left(\frac{[n]_{p, q} t+\alpha}{[n]_{p, q}+\beta}\right) d_{p, q} t,
\end{gathered}
$$

where

$$
\begin{aligned}
b_{n, k}^{p, q}(p, p q t) & =\left[\begin{array}{l}
n \\
k
\end{array}\right]_{p, q}(p t)^{k}(p \ominus p q t)_{p, q}^{n-k}, \\
b_{n, k}^{p, q}(1, x) & =\left[\begin{array}{l}
n \\
k
\end{array}\right]_{p, q} p^{[k(k-1)-n(n-1)] / 2} x^{k}(1 \ominus x)_{p, q}^{n-k} .
\end{aligned}
$$

This type of generalization may be useful because of its flexibility and approximation behaviour may differ for different values of $\alpha$ and $\beta$. For $\alpha=\beta=0$, we observe that these operators reduce to the one given in [10].

Lemma 1 (see [28]). For $m \geqslant 1, e_{i}=t^{i}(i=0,1,2, \ldots)$,

$$
\begin{aligned}
U_{n, m}^{p, q}(x) & :=B_{n, p, q}\left(e_{m}, x\right) \\
& =\sum_{k=0}^{n} b_{n, k}^{p, q}(1, x)\left(\frac{p^{n-k}[k]_{p, q}}{[n]_{p, q}}\right)^{m},
\end{aligned}
$$

we have the following recurrence relation:

$$
\begin{aligned}
{[n]_{p, q} U_{n, m+1}^{p, q}(p x)=} & p^{n}(1-p x) D_{p, q}\left[U_{n, m}^{p, q}(x)\right] \\
& +[n]_{p, q} p x U_{n, m}^{p, q}(p x) .
\end{aligned}
$$

Further,

(1)

$$
B_{n, p, q}\left(e_{0}, x\right)=1
$$

$$
B_{n, p, q}\left(e_{1}, x\right)=x
$$

(4)

$$
B_{n, p, q}\left(e_{2}, x\right)=x^{2}+\frac{p^{n-1} x(1-x)}{[n]_{p, q}} \text {, }
$$

$$
\begin{aligned}
& B_{n, p, q}\left(e_{3}, x\right)=x^{3}+\frac{p^{n-1} x^{2}(1-x)}{[n]_{p, q}} \\
& +\frac{2[n]_{p, q} p^{n-2} x^{2}(1-x)+p^{2 n-2} x(1-x)\left(1-2 x p^{-1}\right)}{[n]_{p, q}^{2}},
\end{aligned}
$$


(5)

$$
\begin{aligned}
& B_{n, p, q}\left(e_{4}, x\right)=x^{4}+\frac{x^{3}(1-x) p^{n-3}\left(p^{2}+2 p+3\right)}{[n]_{p, q}} \\
& +\frac{x^{2}(1-x) p^{2 n-2}\left\{1-2 x p^{-1}+p^{-2}\left(2-3 x p^{-1}\right)(p+2)\right\}}{[n]_{p, q}^{2}} \\
& +\frac{x(1-x) p^{3 n-4}\left(p-4 x p^{-1}-2 x+6 x^{2} p^{-2}\right)}{[n]_{p, q}^{3}} .
\end{aligned}
$$

Lemma 2. For $x \in[0,1], 0<q<p \leqslant 1$, we have

$$
D_{n, \alpha, \beta}^{p, q}(1, x)=1,
$$

$$
D_{n, \alpha, \beta}^{p, q}(t, x)=\frac{p^{n}[n]_{p, q}+\alpha[n+2]_{p, q}+q[n]_{p, q}^{2} x}{\left([n]_{p, q}+\beta\right)[n+2]_{p, q}},
$$

(3)

$$
\begin{aligned}
& D_{n, \alpha, \beta}^{p, q}\left(t^{2}, x\right)=\left\{p^{2 n}[2]_{p, q}[n]_{p, q}^{2}\right. \\
& +\alpha^{2}[n+2]_{p, q}[n+3]_{p, q}+2 \alpha p^{n}[n]_{p, q}[n+3]_{p, q} \\
& +\left\{p^{n} q(p+2 q)[n]_{p, q}^{3}+2 \alpha q[n]_{p, q}^{2}[n+3]_{p, q}\right\} x \\
& \left.+q^{3}[n]_{p, q}^{3}\left\{x^{2}[n]_{p, q}+p^{n-1} x(1-x)\right\}\right\} \\
& +\left(\left([n]_{p, q}+\beta\right)^{2}[n+2]_{p, q}[n+3]_{p, q}\right)^{-1},
\end{aligned}
$$

(4)

$$
\begin{aligned}
& D_{n, \alpha, \beta}^{p, q}\left(t^{3}, x\right)=\left\{p^{3 n}[n]_{p, q}^{3}\left(p^{3}+2 p^{2} q+2 p q^{2}+q^{3}\right)\right. \\
& +3 \alpha p^{2 n}[2]_{p, q}[n]_{p, q}^{2}[n+4]_{p, q}+3 \alpha^{2} p^{n}[n]_{p, q}[n \\
& +3]_{p, q}[n+4]_{p, q}+\alpha^{3}[n+2]_{p, q}[n+3]_{p, q}[n+4]_{p, q} \\
& +\left\{p^{2 n}\left(4 p q^{3}+p^{3} q+3 p^{2} q^{2}+3 q^{4}\right)[n]_{p, q}^{4}\right. \\
& +3 \alpha p^{n}\left(p q+2 q^{2}\right)[n]_{p, q}^{3}[n+4]_{p, q} \\
& \left.+3 \alpha^{2} q[n]_{p, q}^{2}[n+3]_{p, q}[n+4]_{p, q}\right\} x \\
& +\left\{p^{n}\left(2 p q^{4}+p^{2} q^{3}+3 q^{5}\right)[n]_{p, q}^{4}\right. \\
& \left.+3 \alpha q^{3}[n]_{p, q}^{3}[n+4]_{p, q}\right\}\left\{x^{2}[n]_{p, q}\right. \\
& \left.+p^{n-1} x(1-x)\right\}+q^{6}[n]_{p, q}^{4}\left\{x^{3}[n]_{p, q}^{2}\right. \\
& +p^{n-1} x^{2}(1-x)[n]_{p, q}+2[n]_{p, q} p^{n-2} x^{2}(1-x) \\
& \left.\left.+p^{2 n-2} x(1-x)\left(1-2 x p^{-1}\right)\right\}\right\}\left(\left([n]_{p, q}+\beta\right)^{3}[n\right. \\
& \left.+2]_{p, q}[n+3]_{p, q}[n+4]_{p, q}\right)^{-1},
\end{aligned}
$$

Proceeding along the lines of [[10], Lemma 3.1] and using the linearity property of the operators (11), the result follows immediately. We omit the details.

Lemma 3. Denote

$$
\begin{gathered}
\mu_{n, \alpha, \beta, m}^{p, q}(x)=[n+1]_{p, q} \sum_{k=0}^{n} p^{-\left(n^{2}+3 n-k^{2}-k\right) / 2} b_{n, k}^{p, q}(1, x) \\
\cdot \int_{0}^{1} b_{n, k}^{p, q}(p, p q t)\left(\frac{[n]_{p, q} t+\alpha}{[n]_{p, q}+\beta}-x\right)^{m} d_{p, q} t .
\end{gathered}
$$


Then, the central moments are given as follows:

(1)

$$
\begin{aligned}
& \mu_{n, \alpha, \beta, 1}^{p, q}(x) \\
& =\frac{\left\{q[n]_{p, q}^{2}-\left([n]_{p, q}+\beta\right)[n+2]_{p, q}\right\} x+p^{n}[n]_{p, q}+\alpha[n+2]_{p, q}}{\left([n]_{p, q}+\beta\right)[n+2]_{p, q}},
\end{aligned}
$$

$$
\begin{aligned}
& \mu_{n, \alpha, \beta, 2}^{p, q}(x)=\left\{p^{2 n}[2]_{p, q}[n]_{p, q}^{2}+\alpha^{2}[n+2]_{p, q}[n\right. \\
& +3]_{p, q}+2 \alpha p^{n}[n]_{p, q}[n+3]_{p, q}+x\left(p^{n} q(p+2 q)\right. \\
& \quad \cdot[n]_{p, q}^{3}+2 \alpha q[n]_{p, q}^{2}[n+3]_{p, q}+p^{n-1} q^{3}[n]_{p, q}^{3} \\
& \quad-2\left([n]_{p, q} p^{n}+\alpha[n+2]_{p, q}\right)\left([n]_{p, q}+\beta\right) \\
& \left.\quad \cdot[n+3]_{p, q}\right)+x^{2}\left(q^{3}[n]_{p, q}^{4}-p^{n-1} q^{3}[n]_{p, q}^{3}\right. \\
& +\left([n]_{p, q}+\beta\right)^{2}[n+2]_{p, q}[n+3]_{p, q}-2 q[n]_{p, q}^{2} \\
& \left.\left.\quad \cdot\left([n]_{p, q}+\beta\right)[n+3]_{p, q}\right)\right\}\left(\left([n]_{p, q}+\beta\right)^{2}[n+2]_{p, q}[n\right. \\
& \left.+3]_{p, q}\right)^{-1}
\end{aligned}
$$

$$
\begin{aligned}
& \mu_{n, \alpha, \beta, 3}^{p, q}(x)=D_{n, \alpha, \beta}^{p, q}\left(t^{3}, x\right)-3 x D_{n, \alpha, \beta}^{p, q}\left(t^{2}, x\right) \\
& +3 x^{2} D_{n, \alpha, \beta}^{p, q}(t, x)-x^{3} D_{n, \alpha, \beta}^{p, q}(1, x)=\left\{p^{3 n}[n]_{p, q}^{3}\right. \\
& \cdot\left(p^{3}+2 p^{2} q+2 p q^{2}+q^{3}\right)+3 \alpha p^{2 n}[2]_{p, q}[n]_{p, q}^{2}[n \\
& +4]_{p, q}+3 \alpha^{2} p^{n}[n]_{p, q}[n+3]_{p, q}[n+4]_{p, q}+\alpha^{3}[n \\
& +2]_{p, q}[n+3]_{p, q}[n+4]_{p, q} \\
& +\left\{p^{2 n}\left(4 p q^{3}+p^{3} q+3 p^{2} q^{2}+3 q^{4}\right)[n]_{p, q}^{4}\right. \\
& +3 \alpha p^{n}\left(p q+2 q^{2}\right)[n]_{p, q}^{3}[n+4]_{p, q} \\
& \left.+3 \alpha^{2} q[n]_{p, q}^{2}[n+3]_{p, q}[n+4]_{p, q}\right\} x \\
& +\left\{p^{n}\left(2 p q^{4}+p^{2} q^{3}+3 q^{5}\right)[n]_{p, q}^{4}\right. \\
& \left.+3 \alpha q^{3}[n]_{p, q}^{3}[n+4]_{p, q}\right\}\left\{x^{2}[n]_{p, q}\right. \\
& \left.+p^{n-1} x(1-x)\right\}+q^{6}[n]_{p, q}^{4}\left\{x^{3}[n]_{p, q}^{2}\right. \\
& +p^{n-1} x^{2}(1-x)[n]_{p, q}+2[n]_{p, q} p^{n-2} x^{2}(1-x) \\
& \left.\left.+p^{2 n-2} x(1-x)\left(1-2 x p^{-1}\right)\right\}\right\}\left(\left([n]_{p, q}+\beta\right)^{3}[n\right. \\
& \left.+2]_{p, q}[n+3]_{p, q}[n+4]_{p, q}\right)^{-1}-3 x\left\{p^{2 n}[2]_{p, q}\right. \\
& \cdot[n]_{p, q}^{2}+\alpha^{2}[n+2]_{p, q}[n+3]_{p, q}+2 \alpha p^{n}[n]_{p, q}[n \\
& +3]_{p, q}+\left\{p^{n} q(p+2 q)[n]_{p, q}^{3}\right.
\end{aligned}
$$

$$
\begin{aligned}
& \mu_{n, \alpha, \beta, 4}^{p, q}(x)=D_{n, \alpha, \beta}^{p, q}\left(t^{4}, x\right)-4 x D_{n, \alpha, \beta}^{p, q}\left(t^{3}, x\right) \\
& +6 x^{2} D_{n, \alpha, \beta}^{p, q}\left(t^{2}, x\right)-4 x^{3} D_{n, \alpha, \beta}^{p, q}(t, x) \\
& +x^{4} D_{n, \alpha, \beta}^{p, q}(1, x)=\left\{p ^ { 4 n } [ n ] _ { p , q } ^ { 4 } \left(p^{6}+3 p^{5} q+5 p^{4} q^{2}\right.\right. \\
& \left.+6 p^{3} q^{3}+5 p^{2} q^{4}+3 p q^{5}+q^{6}\right)+4 \alpha[n]_{p, q}^{3}[n \\
& +5]_{p, q} p^{3 n}\left(p^{3}+2 p^{2} q+2 p q^{2}+q^{3}\right) \\
& +6 \alpha^{2} p^{2 n}[2]_{p, q}[n]_{p, q}^{2}[n+4]_{p, q}[n+5]_{p, q} \\
& +4 \alpha^{3} p^{n}[n]_{p, q}[n+3]_{p, q}[n+4]_{p, q}[n+5]_{p, q} \\
& +\alpha^{4}[n+2]_{p, q}[n+3]_{p, q}[n+4]_{p, q}[n+5]_{p, q} \\
& +\left\{p ^ { 3 n } \left(12 p^{3} q^{4}+12 p^{2} q^{5}+9 p q^{6}+4 q^{7}+8 p^{4} q^{3}\right.\right. \\
& \left.+p^{6} q+4 p^{5} q^{2}\right)[n]_{p, q}^{5}+4 \alpha p^{2 n}\left(4 p q^{3}+p^{3} q\right. \\
& \left.+3 p^{2} q^{2}+3 q^{4}\right)[n]_{p, q}^{4}[n+5]_{p, q}+6 \alpha^{2} p^{n}(p q \\
& \left.+2 q^{2}\right)[n]_{p, q}^{3}[n+4]_{p, q}[n+5]_{p, q}+4 \alpha^{3} q[n]_{p, q}^{2}[n \\
& \left.+3]_{p, q}[n+4]_{p, q}[n+5]_{p, q}\right\} x+\left\{p ^ { 2 n } \left(9 p q^{7}\right.\right. \\
& \left.+7 p^{3} q^{5}+9 p^{2} q^{6}+6 q^{8}+3 p^{4} q^{4}+p^{5} q^{3}\right)[n]_{p, q}^{5} \\
& \left.+4 \alpha p^{n}\left(2 p q^{4}+p^{2} q^{3}+3 q^{5}\right)[n]_{p, q}^{4}[n+5]_{p, q}\right\} \\
& \cdot\left\{x^{2}[n]_{p, q}+p^{n-1} x(1-x)\right\}+\left\{p ^ { n } \left(3 p q^{8}+2 p^{2} q^{7}\right.\right. \\
& \left.\left.+p^{3} q^{6}+4 q^{9}\right)[n]_{p, q}^{5}+4 \alpha q^{6}[n]_{p, q}^{4}[n+5]_{p, q}\right\} \\
& \cdot\left\{x^{3}[n]_{p, q}^{2}+p^{n-1} x(1-x)[n]_{p, q}+2 p^{n-2} x^{2}(1\right. \\
& \left.-x)[n]_{p, q}+p^{2 n-2} x(1-x)\left(1-2 x p^{-1}\right)\right\} \\
& +q^{10}[n]_{p, q}^{5}\left\{x^{4}[n]_{p, q}^{3}+x^{3}(1-x) p^{n-3}\left(p^{2}+2 p\right.\right. \\
& +3)[n]_{p, q}^{2}+x^{2}(1-x) p^{2 n-2}\left\{1-2 x p^{-1}\right. \\
& \left.+p^{-2}\left(2-3 x p^{-1}\right)(p+2)\right\}[n]_{p, q}+x(1-x)
\end{aligned}
$$




$$
\begin{aligned}
& \left.\left.\cdot p^{3 n-4}\left(p-4 x p^{-1}-2 x+6 x^{2} p^{-2}\right)\right\}\right\}\left(\left([n]_{p, q}\right.\right. \\
& \left.+\beta)^{4}[n+2]_{p, q}[n+3]_{p, q}[n+4]_{p, q}[n+5]_{p, q}\right)^{-1} \\
& -4 x\left\{p^{3 n}[n]_{p, q}^{3}\left(p^{3}+2 p^{2} q+2 p q^{2}+q^{3}\right)\right. \\
& +3 \alpha p^{2 n}[2]_{p, q}[n]_{p, q}^{2}[n+4]_{p, q}+3 \alpha^{2} p^{n}[n]_{p, q}[n \\
& +3]_{p, q}[n+4]_{p, q}+\alpha^{3}[n+2]_{p, q}[n+3]_{p, q}[n+4]_{p, q} \\
& +\left\{p^{2 n}\left(4 p q^{3}+p^{3} q+3 p^{2} q^{2}+3 q^{4}\right)[n]_{p, q}^{4}\right. \\
& +3 \alpha p^{n}\left(p q+2 q^{2}\right)[n]_{p, q}^{3}[n+4]_{p, q}+3 \alpha^{2} q[n]_{p, q}^{2}[n \\
& \left.+3]_{p, q}[n+4]_{p, q}\right\} x+p^{n}\left(2 p q^{4}+p^{2} q^{3}+3 q^{5}\right) \\
& \cdot[n]_{p, q}^{4}+3 \alpha q^{3}[n]_{p, q}^{3}[n+4]_{p, q}\left\{x^{2}[n]_{p, q}\right. \\
& \left.+p^{n-1} x(1-x)\right\}+q^{6}[n]_{p, q}^{4}\left\{x^{3}[n]_{p, q}^{2}+p^{n-1} x^{2}(1\right. \\
& -x)[n]_{p, q}+2[n]_{p, q} p^{n-2} x^{2}(1-x)+p^{2 n-2} x(1 \\
& \left.\left.-x)\left(1-2 x p^{-1}\right)\right\}\right\}\left(\left([n]_{p, q}+\beta\right)^{3}[n+2]_{p, q}[n\right. \\
& \left.+3]_{p, q}[n+4]_{p, q}\right)^{-1}+6 x^{2}\left\{p^{2 n}[2]_{p, q}[n]_{p, q}^{2}+\alpha^{2}[n\right. \\
& +2]_{p, q}[n+3]_{p, q}+2 \alpha p^{n}[n]_{p, q}[n+3]_{p, q}+\left\{p^{n} q(p\right. \\
& \left.+2 q)[n]_{p, q}^{3}+2 \alpha q[n]_{p, q}^{2}[n+3]_{p, q}\right\} x+q^{3}[n]_{p, q}^{3} \\
& \left.\cdot\left\{x^{2}[n]_{p, q}+p^{n-1} x(1-x)\right\}\right\}\left(\left([n]_{p, q}+\beta\right)^{2}[n\right. \\
& \left.+2]_{p, q}[n+3]_{p, q}\right)^{-1}-4 x^{3}\left\{\left(p^{n}[n]_{p, q}+\alpha[n\right.\right. \\
& \left.\left.+2]_{p, q}+q[n]_{p, q}^{2} x\right)\left(\left([n]_{p, q}+\beta\right)[n+2]_{p, q}\right)^{-1}\right\} \\
& +x^{4}:=f_{2}(n, \alpha, \beta, p, q, x) \text {. }
\end{aligned}
$$

The proof follows immediately by applying Lemma 2.

Remark 4. In order to understand the convergence behaviour of the sequence of $(p, q)$-Durrmeyer operators, let $q=\left(q_{n}\right)$ and $p=\left(p_{n}\right)$, such that $0<q_{n}<p_{n} \leqslant 1$ and $p_{n} \rightarrow 1, q_{n} \rightarrow 1, p_{n}^{n} \rightarrow a, q_{n}^{n} \rightarrow b, 0<b<$ $a \leqslant 1$ for $n$ sufficiently large. With this restriction, we have $\lim _{n \rightarrow \infty}[n]_{p_{n}, q_{n}}=\lim _{n \rightarrow \infty}\left(\left(p_{n}^{n}-q_{n}^{n}\right) /\left(p_{n}-q_{n}\right)\right)=(a-b) /(1-$ 1) $=\infty$.

Remark 5. From Lemma 3 and Remark 4, we have

$$
\begin{aligned}
& \lim _{n \rightarrow \infty} \mu_{n, \alpha, \beta, 3}^{p_{n}, q_{n}}(x)=\lim _{n \rightarrow \infty} f_{1}\left(n, \alpha, \beta, p_{n}, q_{n}, x\right)=0, \\
& \lim _{n \rightarrow \infty} \mu_{n, \alpha, \beta, 4}^{p_{n}, q_{n}}(x)=\lim _{n \rightarrow \infty} f_{1}\left(n, \alpha, \beta, p_{n}, q_{n}, x\right)=0 .
\end{aligned}
$$

\section{Direct Estimates}

Let $W^{2}=\left\{g \in C[0,1]: g^{\prime \prime} \in C[0,1]\right\}$, then $K$-functional is defined as

$$
K_{2}(f, \delta)=\inf \left\{\|f-g\|+\delta\left\|g^{\prime \prime}\right\|: g \in W^{2}\right\},
$$

where $\delta>0$ and norm- $\|\cdot\|$ denotes the uniform norm on $C[0,1]$. Following the acclaimed inequality owing to DeVore and Lorentz [29], there exists a constant $C>0$, such that

$$
K_{2}(f, \delta) \leqslant C \omega_{2}(f, \sqrt{\delta})
$$

where the second-order modulus of smoothness for $f \in$ $C[0,1]$ is defined as

$$
\begin{aligned}
\omega_{2} & (f, \delta) \\
& =\sup _{0<h \leqslant \delta} \sup _{x, x+h \in[0,1]}|f(x+2 h)-2 f(x+h)+f(x)| .
\end{aligned}
$$

The usual modulus of continuity for $f \in C[0,1]$ is defined as

$$
\omega(f, \delta)=\sup _{0<h \leqslant \delta} \sup _{x, x+h \in[0,1]}|f(x+h)-f(x)| .
$$

Our prime result is the subsequent local theorem.

Theorem 6. Let $n>3$ be a natural number and $0<q<p \leqslant 1$. Then, there exists a constant $C>0$, such that

$$
\begin{aligned}
& \left|D_{n, \alpha, \beta}^{p, q}(f, x)-f(x)\right| \\
& \leqslant C \omega_{2}\left(f, \sqrt{\mu_{n, \alpha, \beta, 2}^{p, q}(x)+\left(\mu_{n, \alpha, \beta, 1}^{p, q}(x)\right)^{2}}\right) \\
& \quad+\omega\left(f, \mu_{n, \alpha, \beta, 1}^{p, q}(x)\right),
\end{aligned}
$$

where $f \in C[0,1]$ and $x \in[0,1]$.

Proof. For $f \in C[0,1]$, let

$$
\begin{gathered}
\widetilde{D}_{n, \alpha, \beta}^{p, q}(f, x)=D_{n, \alpha, \beta}^{p, q}(f, x)+f(x) \\
-f\left(\frac{p^{n}[n]_{p, q}+\alpha[n+2]_{p, q}}{\left([n]_{p, q}+\beta\right)[n+2]_{p, q}}\right. \\
\left.+\frac{q[n]_{p, q}^{2} x}{\left([n]_{p, q}+\beta\right)[n+2]_{p, q}}\right)
\end{gathered}
$$

Then, using Lemma 2, we immediately get

$$
\begin{aligned}
\widetilde{D}_{n, \alpha, \beta}^{p, q}(1, x)= & D_{n, \alpha, \beta}^{p, q}(1, x)=1, \\
\widetilde{D}_{n, \alpha, \beta}^{p, q}(t, x)= & D_{n, \alpha, \beta}^{p, q}(t, x)+x \\
& -\frac{p^{n}[n]_{p, q}+\alpha[n+2]_{p, q}}{\left([n]_{p, q}+\beta\right)[n+2]_{p, q}} \\
& +\frac{q[n]_{p, q}^{2} x}{\left([n]_{p, q}+\beta\right)[n+2]_{p, q}}=x .
\end{aligned}
$$


Using Taylor’s formula

$$
g(t)=g(x)+(t-x) g^{\prime}(x)+\int_{x}^{t}(t-u) g^{\prime \prime}(u) d u,
$$

$$
\begin{aligned}
& \widetilde{D}_{n, \alpha, \beta}^{p, q}(g, x)=g(x)+\widetilde{D}_{n, \alpha, \beta}^{p, q}\left(\int_{x}^{t}(t-u) g^{\prime \prime}(u) d u, x\right)=g(x)+D_{n, \alpha, \beta}^{p, q}\left(\int_{x}^{t}(t-u) g^{\prime \prime}(u) d u, x\right) \\
& \quad-\int_{x}^{\left(p^{n}[n]_{p, q}+\alpha[n+2]_{p, q}\right) /\left([n]_{p, q}+\beta\right)[n+2]_{p, q}+q[n]_{p, q}^{2} x /\left([n]_{p, q}+\beta\right)[n+2]_{p, q}}\left(\frac{p^{n}[n]_{p, q}+\alpha[n+2]_{p, q}}{\left([n]_{p, q}+\beta\right)[n+2]_{p, q}}+\frac{q[n]_{p, q}^{2} x}{\left([n]_{p, q}+\beta\right)[n+2]_{p, q}}-u\right) \\
& \quad \cdot g^{\prime \prime}(u) d u .
\end{aligned}
$$

we obtain

Thus,

$$
\begin{aligned}
& \left|\widetilde{D}_{n, \alpha, \beta}^{p, q}(g, x)-g(x)\right| \leqslant D_{n, \alpha, \beta}^{p, q}\left(\left|\int_{x}^{t}\right| t-u|| g^{\prime \prime}(u)|d u|, x\right) \\
& \quad+\left|\int_{x}^{\left(p^{n}[n]_{p, q}+\alpha[n+2]_{p, q}\right) /\left([n]_{p, q}+\beta\right)[n+2]_{p, q}+q[n]_{p, q}^{2} x /\left([n]_{p, q}+\beta\right)[n+2]_{p, q}}\right| \frac{p^{n}[n]_{p, q}+\alpha[n+2]_{p, q}}{\left([n]_{p, q}+\beta\right)[n+2]_{p, q}}+\frac{q[n]_{p, q}^{2} x}{\left([n]_{p, q}+\beta\right)[n+2]_{p, q}}-u \mid \\
& \quad \cdot\left|g^{\prime \prime}(u)\right| d u \mid \leqslant\left\{\mu_{n, \alpha, \beta, 2}^{p, q}(x)+\left(\mu_{n, \alpha, \beta, 1}^{p, q}(x)\right)^{2}\right\}\left\|g^{\prime \prime}\right\| .
\end{aligned}
$$

Furthermore, for $f \in C[0,1]$, we have $\left\|D_{n, \alpha, \beta}^{p, q} f\right\| \leqslant\|f\|$ and therefore

$$
\begin{gathered}
\left|\widetilde{D}_{n, \alpha, \beta}^{p, q}(f, x)\right| \leqslant\left|D_{n, \alpha, \beta}^{p, q}(f, x)\right|+|f(x)| \\
+\mid f\left(\frac{p^{n}[n]_{p, q}+\alpha[n+2]_{p, q}}{\left([n]_{p, q}+\beta\right)[n+2]_{p, q}}\right. \\
\left.+\frac{q[n]_{p, q}^{2} x}{\left([n]_{p, q}+\beta\right)[n+2]_{p, q}}\right) \mid \leqslant 3\|f\|
\end{gathered}
$$$$
\forall f \in C[0,1] .
$$

Now, for $f \in C[0,1]$ and $g \in W^{2}$, we get

$$
\begin{aligned}
& \left|D_{n, \alpha, \beta}^{p, q}(f, x)-f(x)\right|=\mid \widetilde{D}_{n, \alpha, \beta}^{p, q}(f, x)-f(x) \\
& +f\left(\frac{p^{n}[n]_{p, q}+\alpha[n+2]_{p, q}}{\left([n]_{p, q}+\beta\right)[n+2]_{p, q}}\right. \\
& \left.+\frac{q[n]_{p, q}^{2} x}{\left([n]_{p, q}+\beta\right)[n+2]_{p, q}}\right)-f(x)|\leqslant| \widetilde{D}_{n, \alpha, \beta}^{p, q}(f
\end{aligned}
$$

$$
\begin{aligned}
& -g, x)|+| \widetilde{D}_{n}^{p, q}(g, x)-g(x)|+| g(x)-f(x) \mid \\
& +\mid f\left(\frac{p^{n}[n]_{p, q}+\alpha[n+2]_{p, q}}{\left([n]_{p, q}+\beta\right)[n+2]_{p, q}}\right. \\
& \left.+\frac{q[n]_{p, q}^{2} x}{\left([n]_{p, q}+\beta\right)[n+2]_{p, q}}\right)-f(x) \mid \leqslant 4\|f-g\| \\
& +\left\{\mu_{n, \alpha, \beta, 2}^{p, q}(x)+\left(\mu_{n, \alpha, \beta, 1}^{p, q}(x)\right)^{2}\right\}\left\|g^{\prime \prime}\right\|+\omega(f, \\
& \left.\left|\mu_{n, \alpha, \beta, 1}^{p, q}(x)\right|\right),
\end{aligned}
$$

where we have used (41) and (42).

On taking infimum on the right hand side over all $g \in$ $W^{2}$, we attain

$$
\begin{aligned}
\left|D_{n, \alpha, \beta}^{p, q}(f, x)-f(x)\right| & \\
\leqslant & 4 K_{2}\left(f, \mu_{n, \alpha, \beta, 2}^{p, q}(x)+\left(\mu_{n, \alpha, \beta, 1}^{p, q}(x)\right)^{2}\right) \\
& \quad+\omega\left(f, \mu_{n, \alpha, \beta, 1}^{p, q}(x)\right) .
\end{aligned}
$$




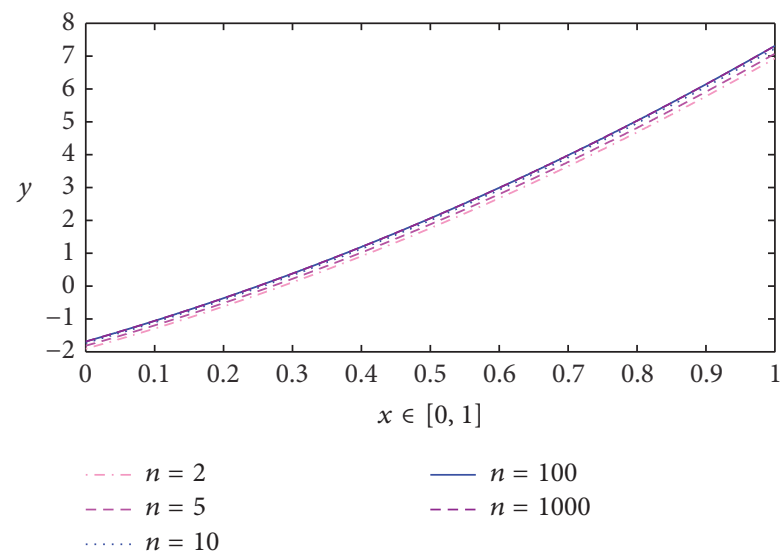

Figure 1: The difference $D_{n, \alpha, \beta}^{0.8,0.3}(f, x)-f(x)$ for $\alpha=5, \beta=20$, and $f(x)=9-6 x-3 x^{2}$.

Lastly, in view of (33), we have

$$
\begin{aligned}
& \left|D_{n, \alpha, \beta}^{p, q}(f, x)-f(x)\right| \\
& \leqslant C \omega_{2}\left(f, \sqrt{\mu_{n, \alpha, \beta, 2}^{p, q}(x)+\left(\mu_{n, \alpha, \beta, 1}^{p, q}(x)\right)^{2}}\right) \\
& \quad+\omega\left(f, \mu_{n, \alpha, \beta, 1}^{p, q}(x)\right) .
\end{aligned}
$$

The theorem is hence proved.

Example 7. We present comparisons and graphs for the convergence of operators $D_{n, \alpha, \beta}^{p, q}(f, x)$ for various values of the parameters $\alpha, \beta, p$, and $q$, such that $0<q<p \leqslant 1$ and $0 \leqslant \alpha \leqslant \beta$. For $x \in[0,1], \alpha=5, \beta=20, p=0.8$, and $q=0.3$, the convergence behaviour of the difference of the operators $D_{n, \alpha, \beta}^{p, q}(f, x)$ to the function $f$, where $f(x)=9-6 x-3 x^{2}$, for various values of $n$, is presented in Figure 1 using MATLAB.

Example 8. The convergence behaviour of the operators $D_{n, \alpha, \beta}^{p, q}(f, x)$ to the function $9-6 x-3 x^{2}$ for $\alpha=0=\beta$ is illustrated in Figure 2. It may be observed that this shows the convergence for the operators defined in [10].

Example 9. We compute the error of approximation for the operators $D_{n, \alpha, \beta}^{p, q}(f, x)$ depending upon the parameters $\alpha$ and $\beta$ at certain points from $[0,1]$ as shown in Table 1 . We consider $n=100, p=0.5, q=0.4$, and $f(x)=9-6 x-3 x^{2}$.

Remark 10. It may be observed from the above example that we may get better convergence depending upon the flexible values of $\alpha$ and $\beta$. This is one of the reasons for discussing operators (11).

We now present the Voronovskaja type asymptotic formula.

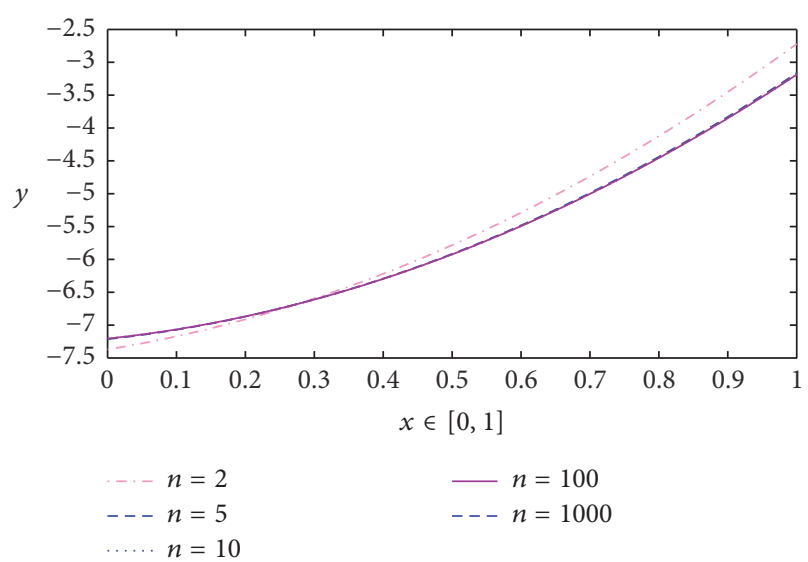

Figure 2: The difference $D_{n, \alpha, \beta}^{0.8,0.3}(f, x)-f(x)$ for $\alpha=0, \beta=0$, and $f(x)=9-6 x-3 x^{2}$.

TABLE 1: Error of approximation.

\begin{tabular}{lcc}
\hline$x$ & $\begin{array}{c}\left|D_{n, \alpha, \beta}^{p, q}(f, x)-f(x)\right| \\
\text { for } \alpha=2, \beta=20\end{array}$ & $\begin{array}{c}\left|D_{n, \alpha, \beta}^{p, q}(f, x)-f(x)\right| \\
\text { for } \alpha=0, \beta=0\end{array}$ \\
\hline 0 & 0.6300 & 3.2640 \\
0.01 & 0.5697 & 3.3624 \\
0.02 & 0.5088 & 3.4612 \\
0.03 & 0.4473 & 3.5603 \\
0.04 & 0.3852 & 3.6599 \\
0.05 & 0.3225 & 3.7598 \\
0.06 & 0.2592 & 3.8601 \\
0.07 & 0.1953 & 3.9608 \\
0.08 & 0.1308 & 4.0619 \\
0.09 & 0.0657 & 4.1634 \\
0.1 & 0 & 4.2652 \\
\hline
\end{tabular}

Theorem 11. Assume $f \in C[0,1]$. If $f^{\prime \prime}$ exists at a point $x \in$ $\left[\alpha /\left([n]_{p_{n}, q_{n}}+\beta\right),\left([n]_{p_{n}, q_{n}}+\alpha\right) /\left([n]_{p_{n}, q_{n}}+\beta\right)\right]$, then under the assumptions of Remark 4, we get

$$
\begin{gathered}
\lim _{n \rightarrow \infty}[n]_{p_{n}, q_{n}}\left(D_{n, \alpha, \beta}^{p_{n}, q_{n}}(f, x)-f(x)\right) \\
=\{a+\alpha-(2 b+\beta) x\} f^{\prime}(x) \\
+\left\{2 a x-(a+b) x^{2}\right\} \frac{f^{\prime \prime}(x)}{2} .
\end{gathered}
$$

Proof. Employing Taylor's expansion of $f$, we get

$$
\begin{aligned}
f(t)= & f(x)+f^{\prime}(x)(t-x)+\frac{f^{\prime \prime}(x)}{2}(t-x)^{2} \\
& +\xi(t, x)(t-x)^{2},
\end{aligned}
$$


where $\xi(t, x) \rightarrow 0$ as $t \rightarrow x$. Applying $D_{n, \alpha, \beta}^{P_{n}, q_{n}}$ to (47), we obtain

$$
\begin{aligned}
D_{n, \alpha, \beta}^{p_{n}, q_{n}}(f, x)-f(x)= & D_{n, \alpha, \beta}^{p_{n}, q_{n}}(t-x, x) f^{\prime}(x) \\
& +D_{n, \alpha, \beta}^{p_{n}, q_{n}}\left((t-x)^{2}, x\right) \frac{f^{\prime \prime}(x)}{2} \\
& +D_{n, \alpha, \beta}^{p_{n}, q_{n}}\left(\xi(t, x)(t-x)^{2}, x\right) .
\end{aligned}
$$

Using Cauchy-Schwarz inequality and (31), we get

$$
\begin{aligned}
& D_{n, \alpha, \beta}^{p_{n}, q_{n}}\left(\xi(t, x)(t-x)^{2}, x\right) \\
& \quad \leqslant \sqrt{D_{n, \alpha, \beta}^{p_{n}, q_{n}}\left(\xi^{2}(t, x), x\right)} \sqrt{D_{n, \alpha, \beta}^{p_{n}, q_{n}}\left((t-x)^{4}, x\right)} .
\end{aligned}
$$

As $\xi^{2}(x, x)=0$ and $\xi^{2}(\cdot, x) \in C[0,1]$, we have

$$
\lim _{n \rightarrow \infty}[n]_{p_{n}, q_{n}} D_{n, \alpha, \beta}^{p_{n}, q_{n}}\left(\xi^{2}(t, x), x\right)=0,
$$

uniformly with respect to $x \in\left[\alpha /\left([n]_{p_{n}, q_{n}}+\beta\right),\left([n]_{p_{n}, q_{n}}+\right.\right.$ $\left.\alpha) /\left([n]_{p_{n}, q_{n}}+\beta\right)\right]$. Therefore, from (49) and (50), we get

$$
\lim _{n \rightarrow \infty}[n]_{p_{n}, q_{n}} D_{n, \alpha, \beta}^{p_{n}, q_{n}}\left(\xi(t, x)(t-x)^{2}, x\right)=0 .
$$

Thus,

$$
\begin{aligned}
\lim _{n \rightarrow \infty} & {[n]_{p_{n}, q_{n}}\left(D_{n, \alpha, \beta}^{p_{n}, q_{n}}(f, x)-f(x)\right)=\lim _{n \rightarrow \infty}[n]_{p_{n}, q_{n}} } \\
\cdot & {\left[D_{n, \alpha, \beta}^{p_{n}, q_{n}}(t-x, x) f^{\prime}(x)\right.} \\
+ & \frac{1}{2} f^{\prime \prime}(x) D_{n, \alpha, \beta}^{p_{n}, q_{n}}\left((t-x)^{2}, x\right) \\
+ & \left.D_{n, \alpha, \beta}^{p_{n}, q_{n}}\left(\xi(t, x)(t-x)^{2}, x\right)\right]=\{a+\alpha \\
& -(2 b+\beta) x\} f^{\prime}(x)+\left\{2 a x-(a+b) x^{2}\right\} \frac{f^{\prime \prime}(x)}{2} .
\end{aligned}
$$

Remark 12. For the $q$-Durrmeyer operators discussed in [22], the recurrence relation was established for $q$-BernsteinDurrmeyer operators (see Theorem 4.3 of [2] and references therein). However, for $(p, q)$-Durrmeyer operators $D_{n, \alpha, \beta}^{p, q}$, it is not analogous and maybe discussed somewhere else.

Remark 13. Let $I=[0,1]$. Then, for $I^{2}=I \times I$, let $C\left(I^{2}\right)$ be the space of all real valued continuous functions on $I^{2}$ equipped with the norm $\|f\|_{I}=\sup _{(x, y) \in I^{2}}|f(x, y)|$.
For $f \in C\left(I^{2}\right)$ and $0<p_{n_{1}}<1,0<p_{n_{2}}<1,0<q_{n_{1}}<1$, and $0<q_{n_{2}}<1$; we construct the bivariate extension of the $(p, q)$-analogue of Durrmeyer-Stancu operators (11) as

$$
\begin{aligned}
& D_{n_{1}, n_{2}, \alpha, \beta}^{p_{n_{1}}, p_{n_{1}}, q_{n_{1}}, q_{n_{2}}}(f, x, y)=\left[n_{1}+1\right]_{p_{n_{1}}, q_{n_{1}}}\left[n_{2}+1\right]_{p_{n_{2}}, q_{n_{2}}} \\
& \cdot \sum_{k_{1}=0}^{n_{1}} \sum_{k_{2}=0}^{n_{2}} p_{n_{1}}^{-\left(n_{1}^{2}+3 n_{1}-k_{1}^{2}-k_{1}\right) / 2} p_{n_{2}}^{-\left(n_{2}^{2}+3 n_{2}-k_{2}^{2}-k_{2}\right) / 2} b_{n_{1}, n_{2}, k_{1}, k_{2}}^{p_{n_{1}}, p_{2}, q_{1}, q_{n_{2}}}(1 \text {, } \\
& x, y) \int_{0}^{1} b_{n_{1}, n_{2}, k_{1}, k_{2}}^{p_{n_{1}}, p_{n_{2}}, q_{n_{1}}, q_{n_{2}}}\left(p_{n_{1}}, p_{n_{2}}, p_{n_{1}} q_{n_{1}} t, p_{n_{2}} q_{n_{2}} s\right) \\
& \cdot f\left(\frac{\left[n_{1}\right]_{p_{n_{1}}, q_{n_{1}}} t+\alpha}{\left[n_{1}\right]_{p_{n_{1}}, q_{n_{1}}}+\beta},\right. \\
& \left.\frac{\left[n_{2}\right]_{p_{n_{2}}, q_{n_{2}}} s+\alpha}{\left[n_{2}\right]_{p_{n_{2}}, q_{n_{2}}}+\beta}\right) d_{p_{n_{1}}, q_{n_{1}}} t d_{p_{n_{2}}, q_{n_{2}}} s
\end{aligned}
$$

where $(x, y) \in I^{2}$ and

$$
\begin{aligned}
& b_{n_{1}, n_{2}, k_{1}, k_{2}}^{p_{n_{1}}, p_{n_{2}}, q_{n_{1}}, q_{n_{2}}}\left(p_{n_{1}}, p_{n_{2}}, p_{n_{1}} q_{n_{1}} t, p_{n_{2}} q_{n_{2}} s\right)=\left[\begin{array}{l}
n_{1} \\
k_{1}
\end{array}\right]_{p_{n_{1}}, q_{n_{1}}} \\
& \cdot\left[\begin{array}{l}
n_{2} \\
k_{2}
\end{array}\right]_{p_{n_{2}}, q_{n_{2}}}\left(p_{n_{1}} t\right)^{k_{1}}\left(p_{n_{2}} s\right)^{k_{2}} \times\left(p_{n_{1}}\right. \\
& \left.\ominus p_{n_{1}} q_{n_{1}} t\right)_{p_{n_{1}}, q_{n_{1}}}^{n_{1}-k_{1}}\left(p_{n_{2}} \ominus p_{n_{2}} q_{n_{2}} s\right)_{p_{n_{2}}, q_{n_{2}}}^{n_{2}-k_{2}} \\
& b_{n_{1}, n_{2}, k_{1}, k_{2}}^{p_{n_{1}}, p_{n_{2}}, q_{n_{1}}, q_{n_{2}}}(1, x, y)=\left[\begin{array}{l}
n_{1} \\
k_{1}
\end{array}\right]_{p_{n_{1}}, q_{n_{1}}}\left[\begin{array}{l}
n_{2} \\
k_{2}
\end{array}\right]_{p_{n_{2}}, q_{n_{2}}} \\
& \cdot p_{n_{1}}^{\left[k_{1}\left(k_{1}-1\right)-n_{1}\left(n_{1}-1\right)\right] / 2} p_{n_{2}}^{\left[k_{2}\left(k_{2}-1\right)-n_{2}\left(n_{2}-1\right)\right] / 2} x^{k_{1}} y^{k_{2}}(1 \\
& \ominus x)_{p_{n_{1}}, q_{n_{1}}}^{n_{1}-k_{1}}(1 \ominus y)_{p_{n_{2}}, q_{n_{2}}}^{n_{2}-k_{2}} .
\end{aligned}
$$

The purpose of this study is to obtain approximation properties of the bivariate generalization of $(p, q)$-BernsteinDurrmeyer operators defined by (11). We may discuss the properties elsewhere.

\section{Conclusion}

In the paper, we have proposed Bernstein-Durrmeyer type operators based on some certain variants. In the case when $\alpha=\beta=0$, our operators reduce to the acclaimed one as defined in [21]. We have derived some approximation properties of Bernstein-Durrmeyer type operators. From those properties, we have estimated its local approximation and Voronoskaja type asymptotic formula. Finally, we have shown some comparisons and illustrative graphs for the convergence of these operators by using the software MATLAB.

\section{Conflicts of Interest}

The authors declare that they have no conflicts of interest. 


\section{Authors' Contributions}

All authors equally contributed to this work.

\section{Acknowledgments}

The second author of this paper is supported by the Research Fund of Hasan Kalyoncu University in 2017.

\section{References}

[1] A. Lupaş, "A $q$-analogue of the bernstein operator," Seminar on numerical and statistical calculus, Nr. 9, University of ClujNapoca, 1987.

[2] A. Aral, V. Gupta, and R. P. Agarwal, Applications of q-Calculus in Operator Theory, Springer, 2013.

[3] V. Gupta and R. P. Agarwal, Convergence Estimates in Approximation Theory, Springer, 2014.

[4] M. Açikgöz, D. Erdal, and S. Araci, "A New approach to qBernoulli numbers and $q$-Bernoulli polynomials related to $q$ Bernstein polynomials," Advances in Difference Equations, vol. 2010, Article ID 951764, 9 pages, 2011.

[5] S. Araci, E. Ağyüz, and M. Acikgoz, "On a q-analog of some numbers and polynomials," Journal of Inequalities and Applications, vol. 2015, article 19, 9 pages, 2015.

[6] V. Kac and P. Cheung, Quantum Calculus, Universitext, Springer, New York, NY, USA, 2002.

[7] M. Mursaleen, K. J. Ansari, and A. Khan, "On ( $p, q)$-analogue of Bernstein operators," Applied Mathematics and Computation, vol. 266, pp. 874-882, 2015.

[8] M. Mursaleen, K. J. Ansari, and A. Khan, "Erratum to 'on $(p, q)$ analogue of Bernstein Operators' [Appl. Math. Comput. 266 (2015) 874-882]," Applied Mathematics and Computation, vol. 278, pp. 70-71, 2016.

[9] M. Mursaleen and F. Khan, "Approximation by Kantorovich type $(p, q)$-Bernstein-Schurer Operators," 2015, https://arxiv .org/abs/1506.02492.

[10] V. Gupta and A. Aral, "Bernstein Durrmeyer operators based on two parameters," Facta Universitatis. Series: Mathematics and Informatics, vol. 31, no. 1, pp. 79-95, 2016.

[11] A. Aral and V. Gupta, “( $p, q)$-type beta functions of second kind," Advances in Operator Theory, vol. 1, no. 1, pp. 134-146, 2016.

[12] S. Araci, U. G. Duran, M. Acikgoz, and H. M. Srivastava, "A certain $(p, q)$-derivative operator and associated divided differences," Journal of Inequalities and Applications, vol. 301, 2016.

[13] V. Gupta, "( $p, q)$-Baskakov-Kantorovich operators," Applied Mathematics and Information Sciences, vol. 10, no. 4, pp. 15511556, 2016.

[14] N. Malik and V. Gupta, "Approximation by $(p, q)$-BaskakovBETa operators," Applied Mathematics and Computation, vol. 293, pp. 49-56, 2017.

[15] G. V. Milovanović, V. Gupta, and N. Malik, “( $p, q)$-Beta functions and applications in approximation," Boletin de la Sociedad Matematica Mexicana. Serie III, pp. 1-19, 2016.

[16] H. Sharma, "On Durrmeyer-type generalization of $(p, q)$-Bernstein operators," Arabian Journal of Mathematics, vol. 5, no. 4, pp. 239-248, 2016.

[17] T. Acar, “( $p, q)$-generalization of Szász-Mirakyan operators," Mathematical Methods in the Applied Sciences, vol. 39, no. 10, pp. 2685-2695, 2016.
[18] Z. Finta, "Approximation properties of $(p, q)$-bernstein type operato," Acta Universitatis Sapientiae, Mathematica, vol. 8, no. 2, pp. 222-232, 2016.

[19] T. Acar, A. Aral, and S. A. Mohiuddine, "On Kantorovich modification of $(p, q)$-Baskakov operators," Journal of Inequalities and Applications, vol. 2016, article 98, 2016.

[20] T. Acar, A. Aral, and S. A. Mohiuddine, "Approximation by bivariate $(p, q)$-Bernstein-Kantorovich operators," Iranian Journal of Science and Technology, Transactions A: Science, pp. 1-8, 2016.

[21] A. Aral, G. Ulusoy, and E. Deníz, "A new construction of SzászMirakyan operators," Numerical Algorithms, pp. 1-14, 2017.

[22] V. Gupta, "Some approximation properties of $q$-Durrmeyer operators," Applied Mathematics and Computation, vol. 197, no. 1, pp. 172-178, 2008.

[23] P. N. Sadjang, "On the $(p, q)$-Gamma and the $(p, q)$-Beta functions," 2015, https://arxiv.org/abs/1506.07394.

[24] P. N. Sadjang, "On the fundamental theorem of $(p, q)$-calculus and some $(p, q)$-Taylor formulas," https://arxiv.org/abs/1309 .3934 .

[25] V. Sahai and S. Yadav, "Representations of two parameter quantum algebras and $p, q$-special functions," Journal of Mathematical Analysis and Applications, vol. 335, no. 1, pp. 268-279, 2007.

[26] Z. Finta and V. Gupta, "Approximation by $q$-Durrmeyer operators," Journal of Applied Mathematics and Computing, vol. 29, no. 1-2, pp. 401-415, 2009.

[27] D. D. Stancu, "Approximation of functions by a new class of linear polynomial operators," Revue Roumaine de Mathématique Pures et Appliquées, vol. 13, pp. 1173-1194, 1968.

[28] V. Gupta, "( $p, q)$-genuine Bernstein Durrmeyer operators," Bollettino dell'Unione Matematica Italiana, vol. 9, no. 3, pp. 399409, 2016.

[29] R. A. DeVore and G. G. Lorentz, Constructive Approximation, Springer, Berlin, Germany, 1993. 


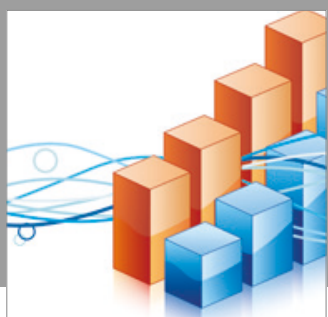

Advances in

Operations Research

vatersals

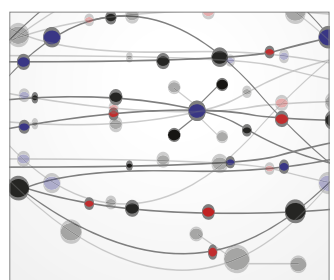

\section{The Scientific} World Journal
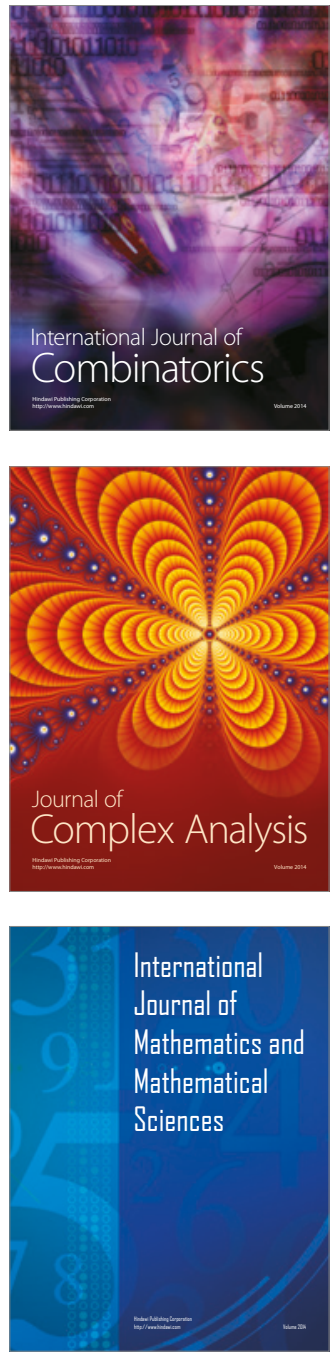
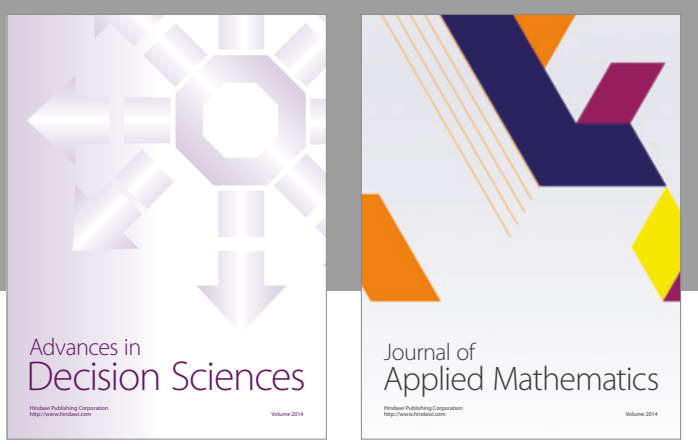

Algebra

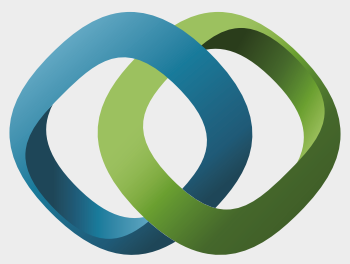

\section{Hindawi}

Submit your manuscripts at

https://www.hindawi.com
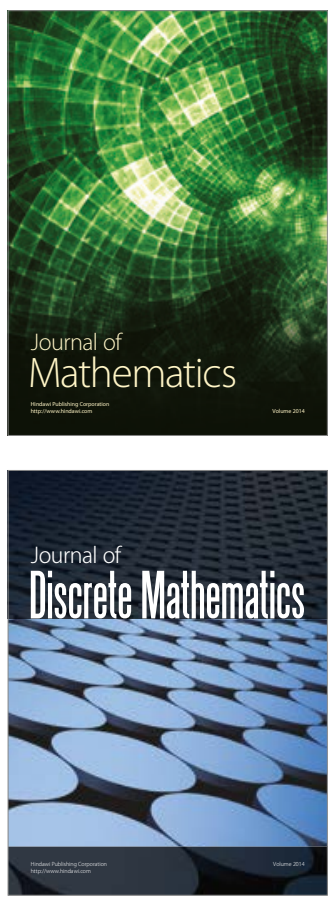

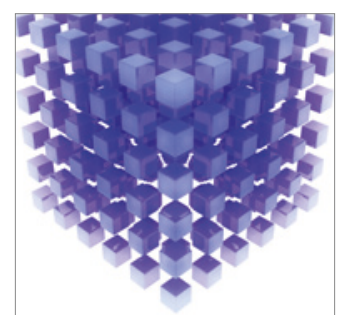

Mathematical Problems in Engineering
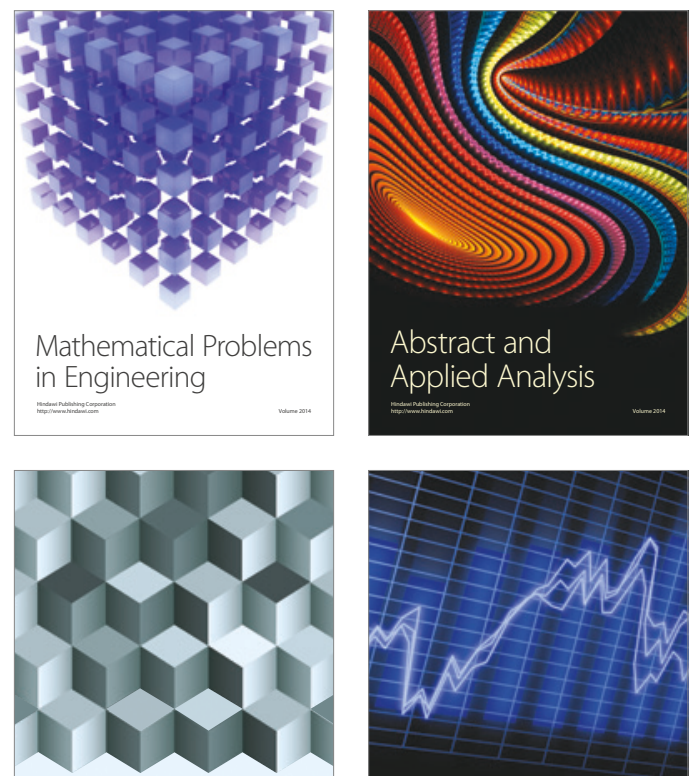

Journal of

Function Spaces

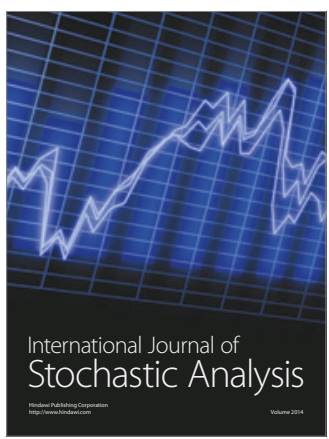

Probability and Statistics
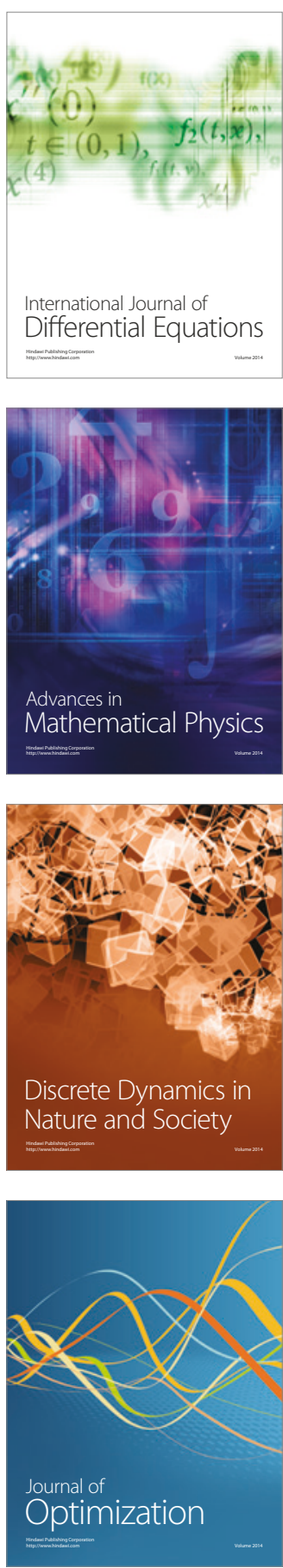